\title{
Kidney Cancer pTX TNM Finding v8
}

National Cancer Institute

\section{Source}

National Cancer Institute. Kidney Cancer PTX TNM Finding v8. NCI Thesaurus. Code C140283.

Kidney cancer in which the primary tumor cannot be assessed. (from AJCC 8th Ed.) 